\title{
ARGAMASSA GEOPOLIMERICA A BASE DE LAMA VERMALHA E METACAULIM PARA REPARO DE ESTRUTURAS DE CONCRETO
}

\author{
CESAR COSTA DO ROSARIO, THÚLIO \\ Graduação em Engenharia Civil \\ Instituto de Tecnologia da Universidade Federal do \\ Pará, FEC - GAEMA - ACGEO \\ Pará, Brasil \\ thuliocostacr@gmail.com
}

\author{
SOUZA PICANÇO, MARCELO \\ Professor Doutor \\ Instituto de Tecnologia da Universidade Federal do \\ Pará, PPGEC - FEC - GAEMA - ACGEO \\ Pará, Brasil \\ marcelosp@ufpa.br
}

\author{
GOMES-PIMENTEL, MAURÍLIO \\ Doutorando em Engenharia Civil \\ Instituto de Tecnologia da Universidade Federal do \\ Pará, PPGEC - FEC - GAEMA - ACGEO \\ Pará, Brasil \\ mauriliogpimentel@gmail.com
}

\author{
GOMES GALIZA-JÚNIOR, JONAS \\ Graduação em Engenharia Civil \\ Instituto de Tecnologia da Universidade Federal do \\ Pará, FEC - GAEMA - ACGEO \\ Pará, Brasil \\ jonasggjunior@gmail.com
}

\author{
NEGRÃO MACÊDO, ALCEBÍADES \\ Professor Doutor \\ Instituto de Tecnologia da Universidade Federal do \\ Pará, PPGEC - FEC - GAEMA - ACGEO \\ Pará, Brasil \\ anmacedo@ufpa.br
}

\section{RESUMO}

O alumínio é cada vez mais usado no mundo em inúmeras funções, porém no seu processo de produção é gerado um resíduo chamado de lama vermelha, o qual não possui destinação, tornando-se então um perigo ambiental devido à grande quantidade armazenada e seu $\mathrm{pH}$ elevado. Entretanto é um material rico em aluminossilicato que o torna propicio para produção de geopolimeros, um material ativado por álcalis. Esse material tem recebido interesses devido a boa resistência ao fogo, corrosão e altas propriedades mecânicas. Nesse estudo investigou se o uso de lama vermelha calcinada como fonte de alumínio e silício para a produção de argamassas geopoliméricas de reparo. Embora não haja parâmetro especificado por norma no Brasil para as proriedades de argamassas de reparo, os resultados sugerem que a argamassa aqui produzida pode servir como reparo de estruturas de concreto, apresentando resistência à compressão após 28 dias de de 40,67 MPa e resistência à flexão de 4,72 MPa. Todos esses resultados mostram que a lama vermelha pode finalmente encontrar uma destinação ambientalmente segura e isso representa uma possível solução para a diminuição da quantidade de resíduo estocado, mitigando riscos ao meio ambiente.

Palavras-chave: Lama vermelha, geopolimero, argamassa de reparo.

\section{ABSTRACT}

Aluminum is increasingly used in the world in various functions, but its production process is waste called red mud, or which has no destination, being used as an environmental hazard due to the large amount stored and its high $\mathrm{pH}$. However, it is a material rich in aluminosilicate that becomes suitable for the production of geopolymers, a material activated by alkalis. This material is of interest due to its good resistance to fire, corrosion and high mechanical properties. In this study he investigated whether the use of calcined red mud as a source of aluminum and silicon for the production of geopolymeric repair mortars. Although there is no parameter specified by norm in Brazil for the properties of repair mortars, the results suggest that the mortar produced here can serve as a repair of concrete structures, presenting compressive strength after 28 days of $40.67 \mathrm{MPa}$ and resistance to flexion of 4.72 MPa. All of these results show that the red mud can finally find an environmentally safe destination and this represents a possible solution for reducing the amount of waste stored, mitigating risks to the environment.

Keyword: Red mud, geopolymer, repair mortar, 


\section{INTRODUÇÃO}

Os recursos econômicos e artificiais que sustentam o bem estar humano foram permitidos graças ao concreto: o ingrediente chave da vida moderna (WEISS et al., 2018), pois possui uma diversificada gama de aplicação, que variam desde estradas a edifícios e pontes, entretanto, o concreto moderno pode se deteriorar em apenas algumas décadas (MEHTA, 1990). Diversos fatores podem causar danos a essas estruturas prejudicando suas propriedades durantes seu período de serviço, como variação excessiva de temperatura, ataque de cloretos e de sulfatos e corrosão de armaduras (LI et al., 2019). Esses danos resultam na necessidades de gastos com inúmeros reparos. Estima-se que os países gastam entre $2 \mathrm{e} 4 \%$ do Produto Interno Bruto (PIB) só com danos relacionados à durabilidade de estruturas de concreto, nos EUA por exemplo, na década de 1990, os gastos com reparos em obras de infraestrutura foi cerca de 300 bilhões de dólares, correspondendo a 5\% do PIB na época (WANG; XU; LIU, 2016).

Alguns materiais são empregados no reparo de estruturas, para aumentar a vida útil do concreto, como os polímeros (LI et al., 2019), todavia destacam-se as argamassas a base de cimento Portland com adições de fibras, argamassas com adições minerais (como sílica ativa e metacaulim) e as argamassas poliméricas (LEHNE; PRESTON, 2018). Porém, esses materiais a base de cimento Portland, exigem um alto custo energético, visto seu atuante papel na poluição atmosférica por meio da emissão de grande quantidade de $\mathrm{CO}_{2}$ durante seu processo de produção, no qual para cada quilograma de cimento Portland produzido, cerca de $93 \%$ em peso de $\mathrm{CO}_{2}$ é emitido para a atmosfera (TEIXEIRA et al., 2019), contribuindo com 8\% das emissões globais de gases do efeito estufa (LEHNE; PRESTON, 2018)). Esforços tem se concentrado na busca em desenvolver materiais alternativos ao cimento Portland para o reparo de estrutura de concreto, que apresentem menores demandas energéticas (LEHNE; PRESTON, 2018), dentre esses materiais, destacam-se os materiais ativados por álcalis, genericamente chamados de geopolímeros.

Geopolímeros são materiais obtidos por meio da reação de um aluminossilicato sólido amorfo e com uma solução aquosa altamente concentrada de hidróxido alcalino ou silicato alcalino (DAVIDOVITS, 1989), esse processo resulta em um material sintético com propriedades aglomerantes, podendo unir agregados para constituição de concretos e argamassas (DUXSON et al., 2007). Os materiais geopoliméricos possuem propriedades promissoras como resistência ao fogo, resistência à corrosão e propriedades mecânicas similar ou superiores ao do cimento Portland (FAHIM HUSEIEN et al., 2017), além de estabilidade química (KUPWADE-PATIL; ALLOUCHE, 2013). Portanto, os materiais geopoliméricos podem ser uma alternativa para uso como material de reparo em estruturas de concreto.

A fonte de aluminossilicato amorfo empregado na síntese de materiais geopoliméricos apresentam um baixo teor de cálcio e geralmente são resíduos ou subprodutos como a cinza volante classe $\mathrm{F}$ e o metacaulim, o que faz aumentar ainda mais o baixo consumo energético desse cimento por conta da diminuição de resíduo de outras indústrias (SINGH; MIDDENDORF, 2020). Já a ativação dos precursores geralmente é realizada com silicato de sódio (Na) ou potássio (K) alcalino, ou hidróxido de sódio ou potássio (DAVIDOVITS, 1989), que são adicionados como solução alcalina com concentração que podem variar de 3 molar a 15 molar (3M a 15M). Logo, materiais ricos em aluminossilicato amorfo são promissores para uso como precursor na síntese de geopolímero, como a lama vermelha (KLAUBER; GRÄFE; POWER, 2011).

A lama vermelha é um rejeito sólido de mineração, gerada durante o processo de beneficiamento do minério bauxita por meio do processo Bayer, estima-se que desde o começo de extração da alumina por esse método até 2007 foram gerados 2,7 bilhões de toneladas desse resíduo e que a quantidade aumenta cerca de 120 milhões de toneladas por ano (POWER; GRÄFE; KLAUBER, 2011). Esse resíduo, no início do século XX, era descartado na natureza. Atualmente, as destinações mais comuns são o armazenamento em lagoas ou o empilhamento a seco, todos esses com riscos de contaminação do solo, águas subterrâneas e consequentemente com riscos a populações em torno desses depósitos. Várias foram as tentativas de encontrar destinação economicamente viável para a lama vermelha, todavia com o avanço dos estudos dos geopolímeros, nota-se que esse resíduo tem características físicas, além de composição química propicia a produção de geopolímeros (KLAUBER; GRÄFE; POWER, 2011). Por exemplo: (YANG et al., 2019), produziram um geopolímero a base de $25 \%$ de lama vermelha e $75 \%$ de cinza volante classe $\mathrm{F}$ como precursores, os resultados sugeririam que a lama vermelha era promissora para a síntese de materiais geopoliméricos, apresentando estabilidade térmica a elevadas temperaturas $\left(600^{\circ} \mathrm{C}\right)$.

Em virtude da crescente necessidade de reparo em estruturas de concreto com utilização de matérias primas mais amigáveis ao meio ambiente, este trabalho caracteriza e avalia o uso da lama vermelha da região Amazônica como fonte de aluminossilicato para a síntese de material geopolimérico, com uma perspectiva para aplicação como argamassas geopoliméricas para reparo de estruturas de concreto. 


\section{MATERIAIS E MÉTODOS}

No presente estudo, como fonte de aluminossilicato para a síntese do material, foi utilizado um metacaulim com massa específica de $2200 \mathrm{~kg} / \mathrm{m}^{3}$, uma lama vermelha com massa específica de $3010 \mathrm{~kg} / \mathrm{m}^{3}$. A análise de fluorescência de raios $\mathrm{X}$ da lama vermelha apresenta uma quantidade de óxido de ferro elevada comparada com o metacaulim (Tabela 1). Além desses materiais foram utilizados um silicato de sódio alcalino $\left(\mathrm{Na}_{2} \mathrm{SiO}_{3}\right)$ com $32 \%$ de $\mathrm{SiO}_{2}$ e $14 \% \mathrm{de}^{2} \mathrm{O}$, apresentando um módulo de sílica (razão molar $\mathrm{SiO}_{2} / \mathrm{Na}_{2} \mathrm{O}$ ) de 2,26 , e densidade a $25^{\circ} \mathrm{C}$ de $1560 \mathrm{~kg} / \mathrm{m}^{3}$. Foi também empregado um hidróxido de sódio em escama $(\mathrm{NaOH})$ com $98 \%$ de pureza, água potável com pH 6,0 e uma areia de origem quartsoza com massa específica de $2650 \mathrm{~kg} / \mathrm{m}^{3}$.

Tabela 1 - Composição química dos materiais em (\%) obtidos por FRX. PF é perda ao fogo.

\begin{tabular}{l|c|c|c|c|c|c|c|c|c|c|c}
\hline \multicolumn{1}{c}{ Material } & $\mathrm{SiO}_{2}$ & $\mathrm{Al}_{2} \mathrm{O}_{3}$ & $\mathrm{Fe}_{2} \mathrm{O}_{3}$ & $\mathrm{MnO}$ & $\mathrm{MgO}$ & $\mathrm{CaO}$ & $\mathrm{Na}_{2} \mathrm{O}$ & $\mathrm{K}_{2} \mathrm{O}$ & $\mathrm{TiO}_{2}$ & $\mathrm{P}_{2} \mathrm{O}_{5}$ & $\mathrm{PF}$ \\
\hline Lama vermelha & 18,30 & 22,60 & 31,00 & 0,16 & $<0,10$ & 1,47 & 11,20 & $<0,10$ & 6,10 & $<0,10$ & 6,94 \\
\hline Metacaulim & 51,54 & 40,50 & 2,80 & - & - & - & 0,08 & 0,18 & - & - & 2,62 \\
\hline
\end{tabular}

Para caracterizar a lama vermelha, primeiramente foi realizado uma análise termogravimétrica sob atmosfera de gás nitrogênio, a temperatura ambiente foi de $25^{\circ} \mathrm{C} \pm 2{ }^{\circ} \mathrm{C}$, foi usada uma massa de $5,8 \mathrm{mg}$. A taxa de aquecimento foi de $10^{\circ} \mathrm{C} / \mathrm{min}$. Além disso, foi realizada análise mineralógica dos materiais por difração de raios $\mathrm{X}$ (DRX), em um difratômetro de feixe divergente equipado com um goniômetro $\theta-\theta$, um tubo de raios $\mathrm{X}$ de $\mathrm{Cu}$ com radiação de $\mathrm{Cu}$ $(\mathrm{K} \alpha 1=1,540598 \AA$ ) e um filtro de Ni K $\beta$. As condições do instrumento foram as seguintes: $40 \mathrm{kV}$ e $40 \mathrm{~mA}$; fenda Soller de $2,5^{\circ}$ (feixe incidente e refratado); faixa angular $(2 \theta)$ de $5^{\circ}$ a $70^{\circ}$. A coleta dos difratogramas foi realizada com passo angular de $0,02^{\circ}$ e tempo por passo de $0,5 \mathrm{~s}^{\circ}$, fenda divergente de $0,6 \mathrm{~mm}$. $\mathrm{O}$ ensaio de granulometria foi feito por difração a laser, o ultrassom foi de 60s, a medida de 60/60/4 e as concentrações de material foram imersas em água.

A análise de microestrutura eletrônica de varredura (MEV) da lama vermelha e do metacaulim foi realizado em um MEV equipado com espectroscopia de energia dispersiva (EDS) foi operado para análises por elétrons retroespalhados. Durante a análise o volume de interação do MEV contém sinais de várias fases, os raios $\mathrm{X}$, por exemplo, geram resultados que permitem a caracterização química dos elementos em determinado ponto da imagem. O MEV foi operado com corrente do feixe de elétrons de $80 \mu \mathrm{A}$, tensão de aceleração constante de $20 \mathrm{kV}$, distância de trabalho foi de $8,5 \mathrm{~mm}$.

\subsection{Preparo das argamassas}

Para a síntese do geopolímero e preparo das argamassas os materiais foram misturados considerando como precursor uma mistura de $30 \%$ de lama vermelha e $70 \%$ de metacaulim, a proporção dos materiais utilizados para a mistura da argamassa está representado na Figura 1A. A mistura foi realizada em um misturador mecânico com capacidade de 5 litros e velocidade de rotação de $140 \pm 5 \mathrm{Rr} / \mathrm{min}$. A solução de silicato de sódio foi adicionada ao misturador junto com o $\mathrm{NaOH}$ e a água, esses materiais foram misturados em um intervalo de tempo de 5 minutos (Figura 1B), após esse intervalo de tempo, o metacaulim com a lama vermelha foram adicionados ao misturador, e misturou-se por 2 minutos, por fim, adicionou-se a areia e misturou-se por mais 3 minutos.

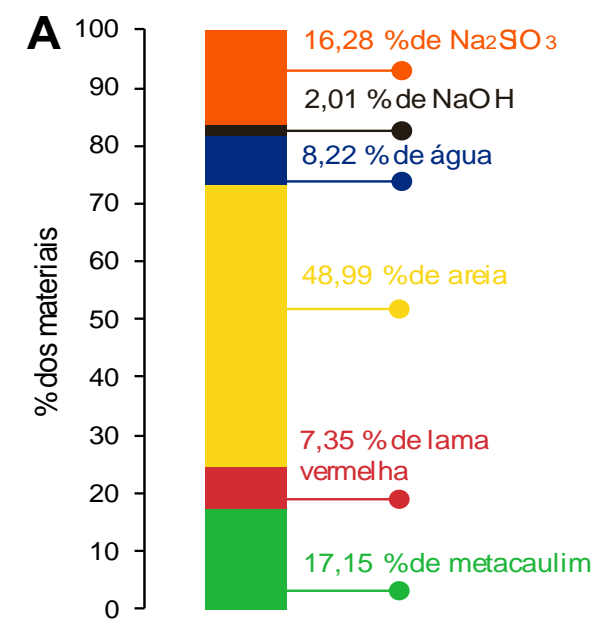

B

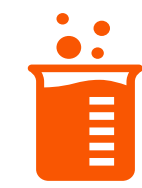

$\mathrm{Na}_{2} \mathrm{SO}_{3}$

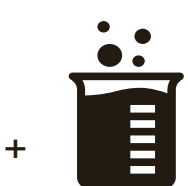

$\mathrm{NaOH}$

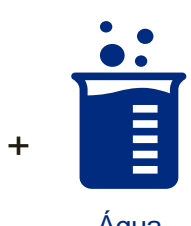

Água

Misturou- $\rightsquigarrow$ os materiais acima ao mesmo tempo em um misturador mecânico com volume de $5 \mathrm{I}$, em velocidade de $140 \pm 5 \mathrm{R} /$ min por 5 minutos
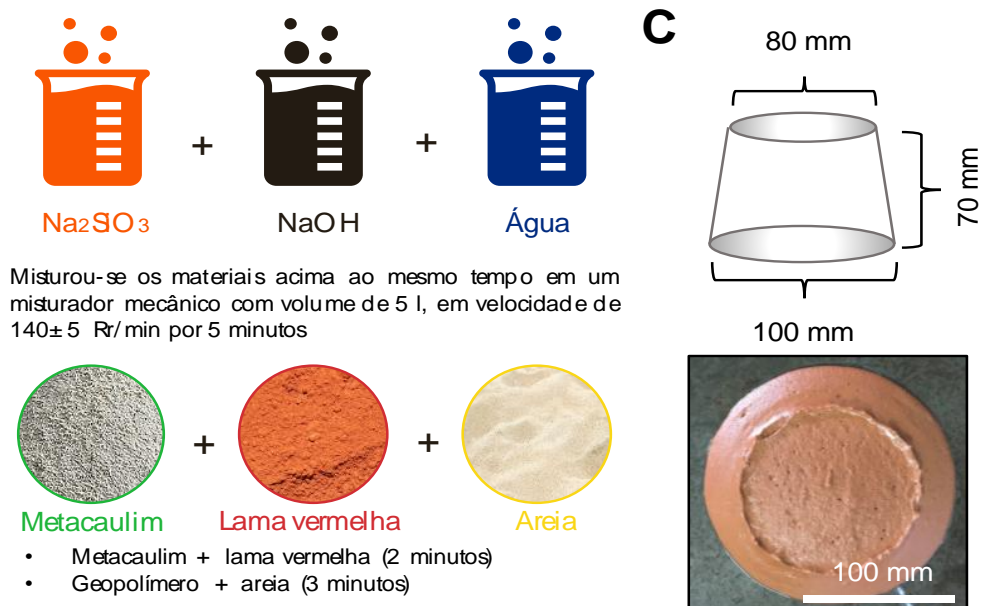

$100 \mathrm{~mm}$

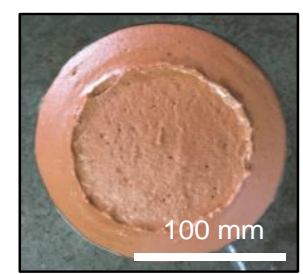

Figura 1: Projeto de fabricação da argamassa geopolimérica. (A) a mistura geral da argamassa considera um total de $24,5 \%$ de material precursor. (B) Os detalhes da mistura revelam um total de tempo de mistura de 10 minutos. (C) consistência da argamassa. 
Embora não seja objeto de estudo do presente trabalho questões reológicas, foi considerada a medida de consistência da argamassa imediatamente após a mistura em um tronco de cone (Figura 1C) para viés de reprodução, não foi dado golpes na mesa de consistência, apenas passado óleo mineral, o material foi adicionado no tronco de cone e após preenchimento, o tronco de cone foi removido permitindo que o material escoasse. Foram retiradas 4 medidas com o paquímetro de precisão, o que permitiu calcular uma média de 166,27 $\mathrm{mm} \pm 1,92 \mathrm{~mm}$.

Após a mistura a argamassa foi adicionada em moldes cilíndricos com dimensões $\varnothing 50 \mathrm{~mm}$ x $100 \mathrm{~mm}$, e prismáticos com dimensões de $40 \mathrm{~mm}$ x $40 \mathrm{~mm}$ x $160 \mathrm{~mm}$ e acondicionadas em uma câmara térmica com temperatura de $30^{\circ} \mathrm{C}$ e umidade relativa de $60 \%$. As amostras cilíndricas foram utilizadas no ensaio de resistência à compressão e absorção total de água de acordo com a NBR 9778 (ABNT, 2005), enquanto as amostras prismática foram submetidas ao ensaio de resistência à tração na flexão. Os ensaios mecânicos foram realizados em um prensa universal, a velocidade do carregamento à compressão foi de $0,25 \mathrm{MPa} / \mathrm{s}$, já a velocidade do ensaio na flexão foi de $0,1 \mathrm{~mm} / \mathrm{min}$. Os ensaios foram realizados na idade de 28 dias. O número de repetições para cada ensaio foi de quatro ( $\mathrm{N}=4)$.

\section{RESULTADOS E DISCUSSÃO}

\subsection{Caracterização dos materiais}

A análise termogravimétrica da lama vermelha revela três fases substanciais de perda de massa (Figura 2A), a primeira está na faixa de $50^{\circ} \mathrm{C}$ a $260^{\circ} \mathrm{C}$ relacionado a perda de água das partículas (MERCURY et al., 2010). A segunda fase está associada ao mineral gibbsita, nessa fase, na temperatura de $270^{\circ} \mathrm{C}$ a $317^{\circ} \mathrm{C}$ ocorre a transformação de $\gamma-\mathrm{Al}(\mathrm{OH})_{3} \mathrm{em} \gamma-$ $\mathrm{AlOOH}$ (ZHU; FANG; LI, 2010) (Equação 1), em seguida, até a temperatura de $680^{\circ} \mathrm{C}$ ocorre a conversão do $\gamma$-AlOOH em $\mathrm{Al}_{2} \mathrm{O}_{3}$ amorfo (Equação 2). A análise termogravimétrica da amostra permitiu quantificar na faixa de temperatura da segunda fase da amostra de lama vermelha um total de 7,96\% de gibbsita $\left(\gamma-\mathrm{Al}(\mathrm{OH})_{3}\right)$. Já a terceira fase relacionada a perda de massa, pode ser associada a perda de espécies voláteis que estão retidas no interior da sodalita (Mercury et al., 2010).

$$
\begin{array}{r}
\gamma-\mathrm{Al}(\mathrm{OH})_{3} \rightarrow \gamma-\mathrm{AlOOH}+\mathrm{H}_{2} \mathrm{O} \\
\gamma-\mathrm{AlOOH} \rightarrow 0,5 \mathrm{Al}_{2} \mathrm{O}_{3}+0,5 \mathrm{H}_{2} \mathrm{O}
\end{array}
$$

(Equação 2)
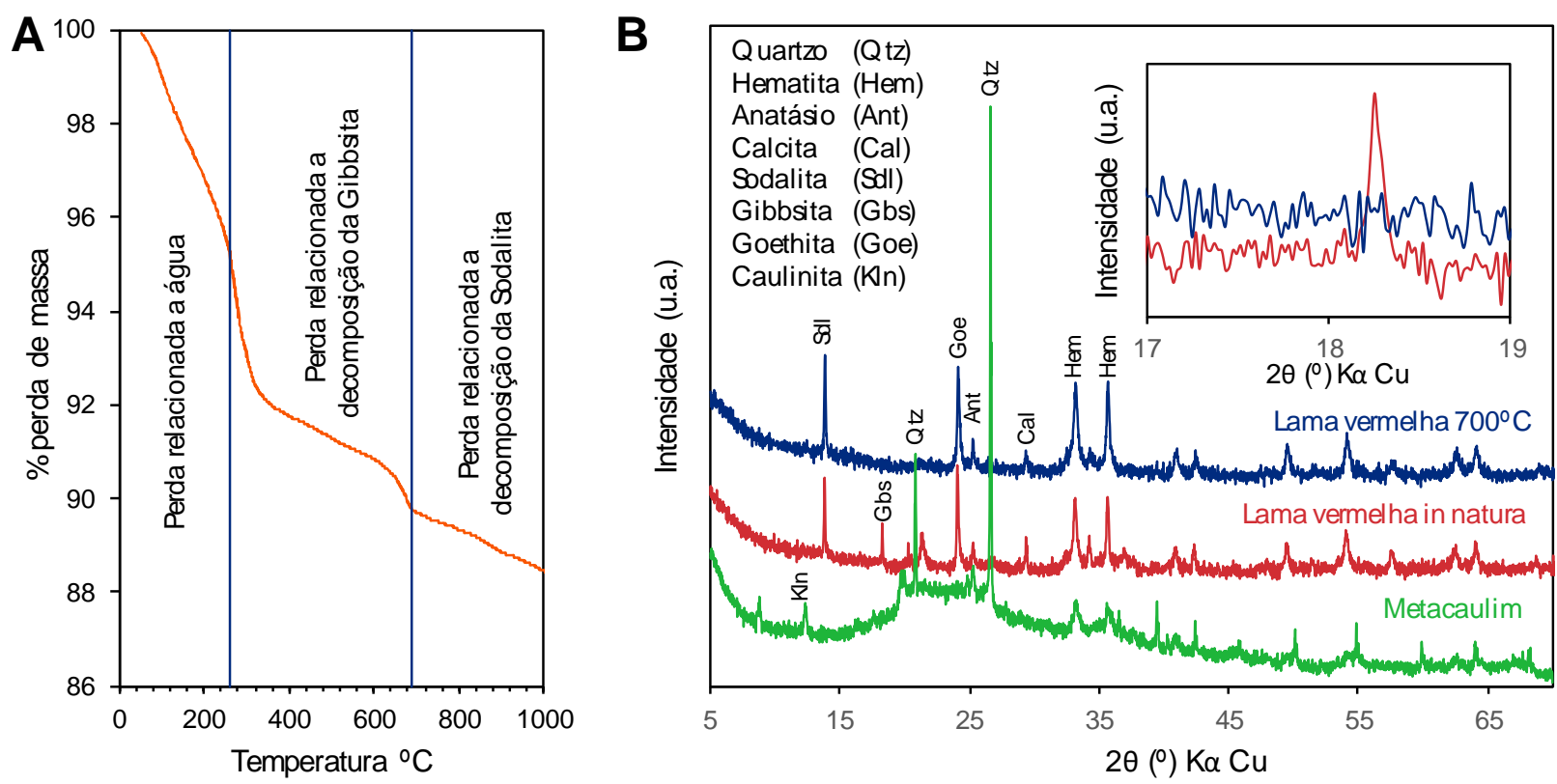

Figura 2: Análise térmica e mineralógica. A análise térmica da lama vermelha revela 3 níveis de decomposição substancial relacionada especialmente a a transformação de fase da água e gibbsita $(\mathbf{A})$. O beneficiamento térmico da lama vermelha desestruturou o mineral gibbsita $(\mathbf{B})$.

A fase amorfa do $\mathrm{Al}_{2} \mathrm{O}_{3}$ é de extrema importância para a síntese geopolimérica (HUANG; HAN, 2011). Por conta disso, a lama vermelha utilizada no presente estudo passou por um beneficiamento térmico antes de ser utilizada na síntese. $\mathrm{O}$ beneficiamento consistiu na calcinação do material em um forno mufla na temperatura de $700^{\circ} \mathrm{C}$ por uma hora. Esse tratamento permitiu uma total conversão da gibbsita presente na lama vermelha in natura para a fase $\mathrm{Al}_{2} \mathrm{O}_{3}$ amorfa 
(imagem ampliada da Figura 2B). A lama vermelha é caracterizada por um diversificado número de fases, já o metacaulim usado para a síntese apresentou apenas caulinita que provavelmente é residual pela queima ineficiente do material, anatásio e quartzo. Além disso, a lama vermelha não apresenta qualquer indício de presença do quartzo, indicando que o $\mathrm{SiO}_{2}$ (Tabela 1) do material pode estar na fase amorfa ou associado a fase sodalita.

A microestrutura eletrônica de varredura dos materiais, permitiu revelar que as partículas de lama vermelha são mais finas que as partículas de metacaulim (Figura 3A e 3C). De fato, a análise granulométrica a laser (Figura 3B) confirmou que a $90 \%$ das partículas de lama vermelha estão abaixo de $29,1 \mu \mathrm{m}$ com o diâmetro médio de $8,5 \mu$ m, já o metacaulim possui $90 \%$ das partículas inferior a $62,2 \mu \mathrm{m}$ e um diâmetro médio de $24,8 \mu \mathrm{m}$. Isso sugere que a lama vermelha apresenta uma superfície específica superior que o metacaulim. A frequência do tamanho das partículas pode ser observada nas imagens de microestrutura (Figura 3A e 3C).

A ampliação da microestrutura da lama vermelha (Figura 3D) permitiu caracterizar uma partícula de aglomerados de hematita, confirmado pela varrimento na linha laranja e representado na Figura 3E. O entorno da partícula é caracterizado por uma composição difusa de sódio (Na), alumínio (Al), silício ( $\mathrm{Si}$ ) e titânio (Ti). O varrimento por EDS da Figura 3C é representado na Figura 3F e 3G, confirmam a frequência relacionada a quantidade de alumínio e silício do metacaulim (Tabela 1). As partículas com coloração laranja mais intensa estão relacionadas a partículas de quartzo, as demais partículas podem estar associada a fase metacaulinita ou caulinita, já que houve evidências de caulinita residual na amostra.
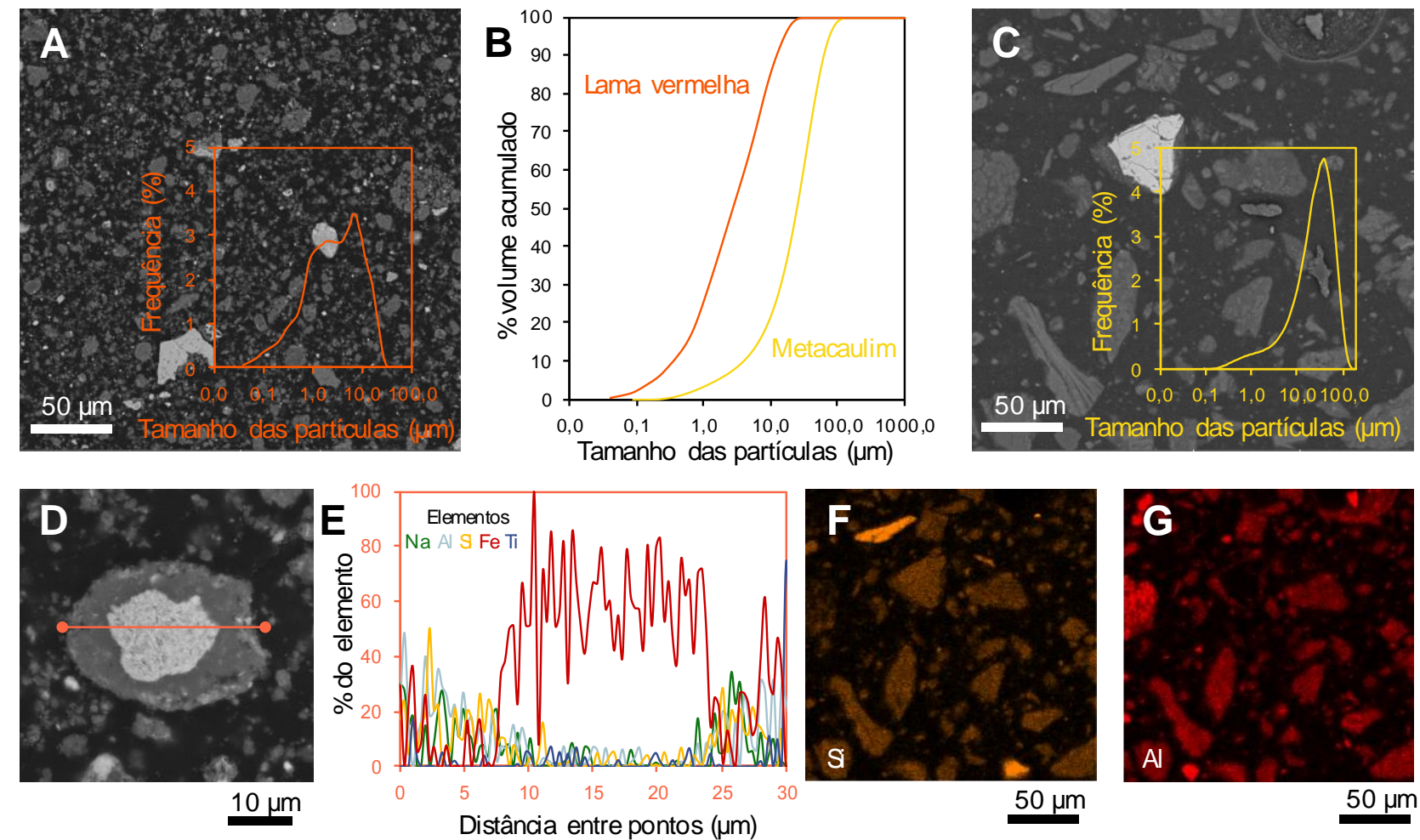

Figura 3: Granulometria e microestrutura dos materiais. (A) MEV da lama vermelha. (B) granulometria a laser dos materiais (lama vermelha calcinada e metacaulim). (C) MEV do metacaulim. (D) ampliação de uma partícula de lama vermelha constituída majoritariamente de Fe, a linha laranja de $\mathbf{D}$ é varrida por EDS e mostrado em E. Um mapa de Si e Al foi realidado na amostra de metacaulim em $\mathbf{C}$ e é mostrado em $\mathbf{F}$ e $\mathbf{G}$ respectivamente.

\subsection{Propriedades da argamassa e implicações para o reparo de estruturas de concreto}

Muitos trabalhos afirmam que materiais geopoliméricos apresentam propriedades mecânicas compatíveis com argamassas convencionais à base de cimento Portland (HU et al., 2008; J. DAVIDOVITS, 2015; PROVIS; DEVENTER, 2009), no entanto para argamassas de reparo somente esta informação não é suficiente, o que gera a necessidade de adequação a parâmetros iniciais de propriedades mecânicas.

Para avaliar o potencial uso da argamassa geopolimérica a base de lama vermelha e metacaulim produzida no presente estudo, foi adotado às respostas dos ensaios de resistência à compressão e resistência à flexão, os valores de resistência 
foram associados as classes de resistência da norma europeia (EN 1504-3, 2006). A supracitada norma estabelece 4 classes de argamassas de reparo, a saber: R1, R2, R3 e R4. Enquanto as classes R1 e R2 são adequadas para reparos não estruturais, as classes R3 e R4 são indicadas para reparo estrutural.

Às respostas à resistência à compressão, permitiram classificar a argamassa produzida no presente estudo na classe R3 (Figura 4). O valor de resistência $(40,67 \mathrm{MPa} \pm 1,69 \mathrm{MPa})$ é superior ao limite estabelecido para a classe R3 ( $\geq 25$ $\mathrm{MPa})$. A resistência à tração na flexão das amostras $(4,72 \mathrm{MPa} \pm 0,81 \mathrm{MPa})$ permite calcular a relação entre resistência à compressão e resistência à tração, obtendo um valor de 11,60\%. Essa relação é similar as argamassas geopoliméricas a base de cinza volante e metacaulim (KRAMAR; ŠAJNA; DUCMAN, 2016) que foram empregadas para reparo de estrutura de concreto armado, e de argamassas de cimento Portland que foram empregadas no reparo de laje de concreto armado.

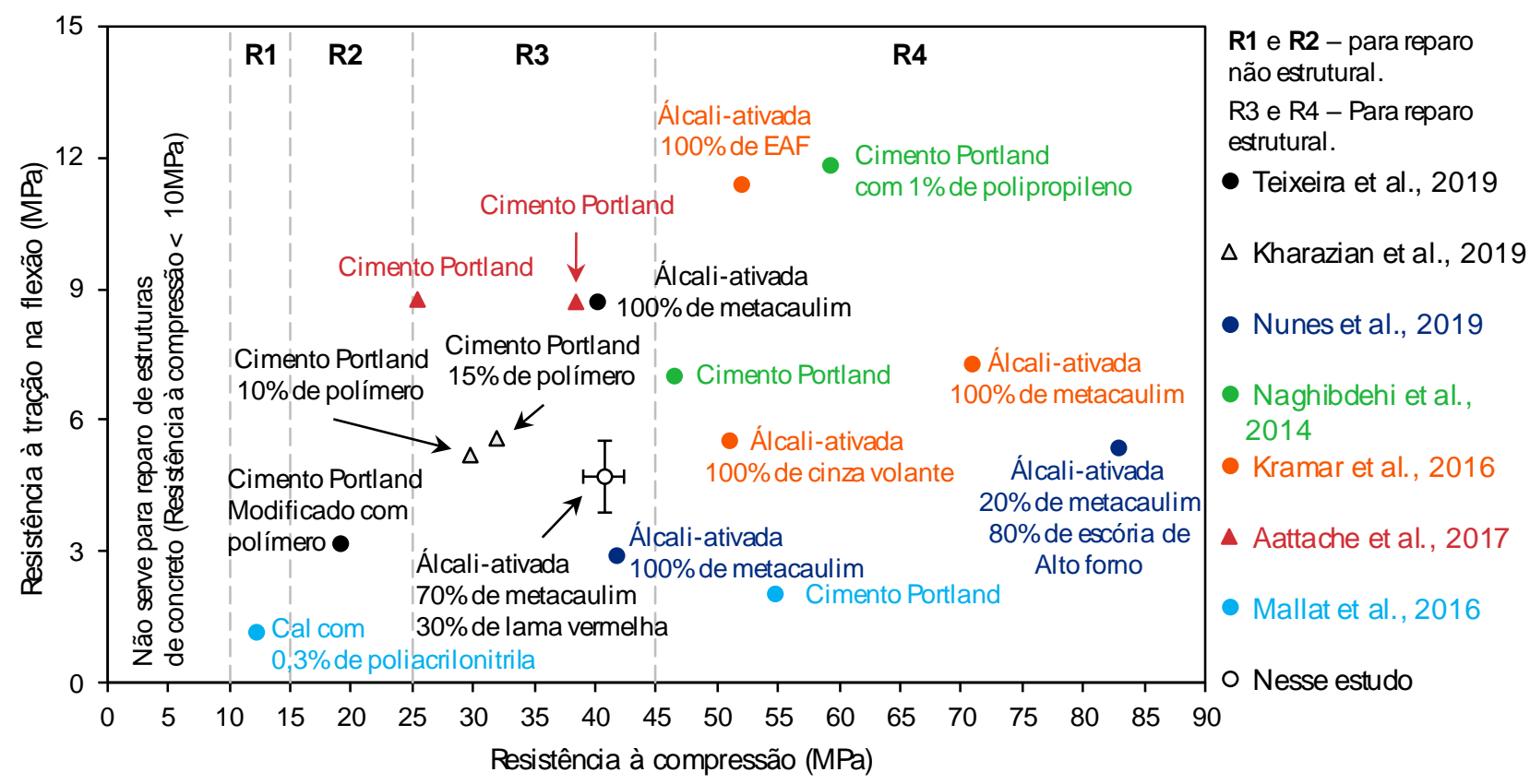

Figura 4: Comportamento mecânico da argamassa geopolimérica a base de lama vermelha e metacaulim. Os valores estão representados como média \pm desvio padrão.

Os resultados de resistência à compressão e a flexão foram compilados com os resultados de argamassas que foram empregada em reparo de estruturas de concreto, e estão representados na Figura 4. Os resultados sugerem que a argamassa produzida no presente estudo tem propriedades similares a uma argamassa álcali ativada que usa metacaulim como precursos (NUNES et al., 2019), e argamassa convencional de cimento Portland (AATTACHE et al., 2017) bem como argamassas a base de cimento Portland modificadas com polímeros (KHARAZIAN et al., 2019). Essa comparação sugere que a argamassa a base de lama vermelha e metacaulim possuem excelentes propriedades mecânicas para reparo de estrutura de concreto armado. Além disso, é possível notar que as propriedades mecânicas dessas argamassas variam de acordo com os materiais empregados no processo de fabricação.

Sabe-se que para realizar o emprego do material relatado nesse trabalho é necessário mais caracterização das propriedades, como módulo de elasticidade e especialmente compatibilidade do substrato (concreto) com a argamassa álcali-ativada. Por exemplo, a EN 1504-3, 2006 também sugere uma classe de argamassa baseada na resistência de aderência. Para que de fato a argamassa desse estudo fosse utilizada em reparos de estruturas, seria necessário o mínimo de 1,5 MPa. Entretanto a força de ligação entre materiais ativados por álcali e compósitos a base de cimento Portland já é bem conhecida na literatura, apresentando perfeita compatibilidade (KRAMAR et al., 2016). Além disso, os resultados apresentados na Figura 4 sugerem que para aumentar a resistência à flexão do material o uso de fibra de polipropileno seria uma alternativa viável (NAGHIBDEHI et al., 2014).

Além disso, Cusson e Mailvaganam, 1996, estabeleceram alguns intervalos para as principais propriedades mecânicas de materiais de reparo, entre os materiais especificados encontra-se a classe de materiais cimentícios no qual a resistência à compressão deve variar entre 20 e $50 \mathrm{MPa}$, resistência à tração entre 2 e $5 \mathrm{MPa}$ e absorção de água de 5 a $15 \%$ em peso. Nesse sentido, fica evidente que a argamassa aqui caracterizada apresenta propriedades mecânicas propícias para o uso em reparo de estruturas, uma vez que a resistência à compressão, à tração e absorção de água foram 
respectivamente 40,67 $\mathrm{MPa}, 4,72 \mathrm{MPa}$ e 14\%. Da mesma forma, o valor da massa específica da argamassa a base de lama vermelha e metacaulim foi de $2070 \mathrm{Kg} / \mathrm{m}^{3}$, sugerindo que o materiail possue um peso inferior ao materiais normalmente empregados no reparo de estruturas de concreto, que são próximos de $2500 \mathrm{Kg} / \mathrm{m}^{3}$ (AATTACHE et al., 2017).

\section{CONCLUSÕES}

Este estudo gera notícias animadoras na tentativa de incorporação do resíduo da bauxita a uma cadeia produtiva, uma vez que os resultados mostraram que a lama vermelha pode servir com fonte de aluminossilicatos para argamassas geopoliméricas de reparo, destacando que a calcinação possibilita melhores condições de síntese do gel geopolimérico, pois a sinterização fornece mais alumínio reativo proveniente da desidroxilação da gibbsita. Isso pode ser percebido por meio das propriedades mecânicas e microestruturais discutidas no trabalho. No entanto, outros estudos devem ser feitos em relação a durabilidade e aplicabilidade para que esse material seja realmente utilizado na construção civil. O que pode implicar em uma potencial desaceleração do aumento no estoque desse resíduo, podendo haver diminuição de riscos ao meio ambiente. Além de tudo isso, a incorporação desse produto no mercado pode produzir uma diminuição dos níveis de $\mathrm{CO}_{2}$ oriundos da indústria do cimento Portland a qual contribui significativamente para o efeito estufa culminando no aquecimento global.

\section{AGRADECIMENTOS}

Os autores são extremamentes gratos ao Laboratório de Materiais Cerâmicos (LACER) da Universidade Federal do Rio Grande do Sul pela análise termogravimétriga da lama vermelha (LV), granulometria a laser e FRX do MK e da LV. Ao Laboratório de Raios X, Análise Termica e Dielétrica do PPGF da UFPA pela análise de DRX, ao Laboratório de Metalografia do Instituto Federal do Pará pela análise de MEV da LV e do MK, a CAPES e a PROPESP-UFPA pelo auxílio em forma de bolsa de iniciação científica e de pós-graduação e aos integrantes do grupo Avanços na Ciência do Geopolímero da UFPA pelos constantes debates e constribuições para a conclusão desse artigo.

\section{REFERÊNCIAS}

AATTACHE, A.; SOLTANI, R.; MAHI, A. Investigations for properties improvement of recycled PE polymer particles-reinforced mortars for repair practice. Construction and Building Materials, v. 146, p. 603-614, 2017.

DAVIDOVITS, J. Geopolymers and geopolymeric materials. Journal of Thermal Analysis, 1989.

DUXSON, P. et al. Geopolymer technology: The current state of the art. Journal of Materials Science, v. 42, n. 9, p. 2917-2933, 2007.

ESTONIAN CENTRE FOR STANDARDISATION. Products and systems for the protection and repair of concrete structures - Definitions, requirements, quality control and evaluation of conformity - Part 3: Structural and nonstructural repair. EN 1504-3, 2006.

FAHIM HUSEIEN, G. et al. Geopolymer mortars as sustainable repair material: A comprehensive review. Renewable and Sustainable Energy Reviews, v. 80, n. May, p. 54-74, 2017.

HUANG, Y.; HAN, M. The influence of $\alpha$-Al2O3 addition on microstructure, mechanical and formaldehyde adsorption properties of fly ash-based geopolymer products. Journal of Hazardous Materials, 2011.

KHARAZIAN, H. A. et al. Effect of water-based acrylic copolymer on void systems of cementitious repair mortar. Case Studies in Construction Materials, v. 11, p. e00261, 2019.

KLAUBER, C.; GRÄFE, M.; POWER, G. Bauxite residue issues: II. options for residue utilization. Hydrometallurgy, v. 108, n. 1-2, p. 11-32, 2011.

KRAMAR, S.; ŠAJNA, A.; DUCMAN, V. Assessment of alkali activated mortars based on different precursors with regard to their suitability for concrete repair. Construction and Building Materials, v. 124, p. 937-944, 2016.

KUPWADE-PATIL, K; ALLOUCHE, E. N. Impact of alkali silica reaction on fly ash-based geopolymer concrete. Journal of Materials in Civil Engineering, v. 25, n. 1, p. 131-139, 2013. 
LEHNE, J.; PRESTON, F. Making Concrete Change; Innovation in Low-carbon Cement and Concrete. Chatham House Report, p. 1-122, 2018.

LI, ZHE et al. Use of a novel electro-magnetic apparatus to monitor corrosion of reinforced bar in concrete. Sensors and Actuators, A: Physical, v. 286, p. 14-27, 2019.

MALlAT, A.; ALLICHE, A. Mechanical investigation of two fiber-reinforced repair mortars and the repaired system. Construction and Building Materials, v. 25, n. 4, p. 1587-1595, 2011.

MERCURY, J. M. R. et al. Estudo do comportamento térmico e propriedades físico-mecânicas da lama vermelha. Revista Materia, v. 15, n. 3, p. 445-460, 2010.

NAGHIBDEHI, M. G.; SHARBATDAR, M. K.; MASTALI, M. Repairing reinforced concrete slabs using composite layers. Materials and Design, v. 58, p. 136-144, 2014.

NUNES, V. A.; BORGES, P. H. R.; ZANOTTI, C. Mechanical compatibility and adhesion between alkali-activated repair mortars and Portland cement concrete substrate. Construction and Building Materials, v. 215, p. 569-581, 2019.

POWER, G.; GRÄFE, M.; KLAUBER, C. Bauxite residue issues: I. Current management, disposal and storage practices. Hydrometallurgy, v. 108, n. 1-2, p. 33-45, 2011.

SINGH, N. B.; MIDDENDORF, B. Geopolymers as an alternative to Portland cement : An overview. Construction and Building Materials, v. 237, p. 117455, 2020.

TEIXEIRA, O. G. et al. Mortar type influence on mechanical performance of repaired reinforced concrete beams. Construction and Building Materials, v. 217, p. 372-383, 2019.

WANG, B.; XU, S.; LIU, F. Evaluation of tensile bonding strength between UHTCC repair materials and concrete substrate. Construction and Building Materials, v. 112, p. 595-606, 2016.

WEISS, D. J. et al. A global map of travel time to cities to assess inequalities in accessibility in 2015. Nature, v. 553 , n. 7688, p. 333-336, 2018.

YANG, Z. et al. Preparation of a geopolymer from red mud slurry and class F fly ash and its behavior at elevated temperatures. Construction and Building Materials, v. 221, p. 308-317, 2019.

ZHU, B.; FANG, B.; LI, X. Dehydration reactions and kinetic parameters of gibbsite. Ceramics International, v. 36, n. 8, p. 2493-2498, 2010. 